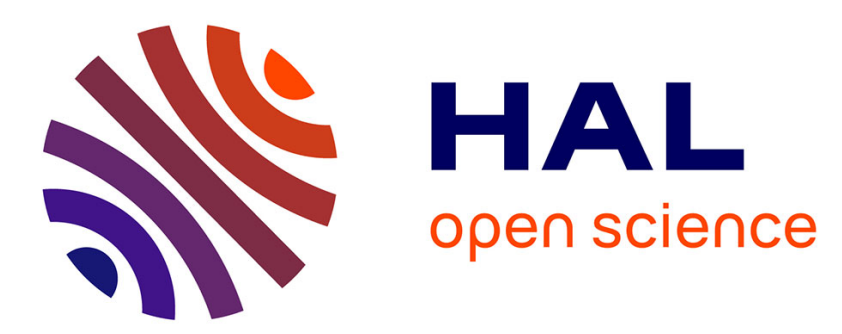

\title{
On the Self-Organization of Mobile Agents to Ensure Dynamic Multi-level Coverage in Sensor Networks
}

\author{
Younes Regragui, Najem Moussa
}

\section{To cite this version:}

Younes Regragui, Najem Moussa. On the Self-Organization of Mobile Agents to Ensure Dynamic Multi-level Coverage in Sensor Networks. The 2nd International Conference on Networking, Information Systems \& Security, Mar 2019, Rabat, Morocco. 10.1145/3320326.3320341 . hal-02494854

\section{HAL Id: hal-02494854 \\ https://hal.science/hal-02494854}

Submitted on 29 Feb 2020

HAL is a multi-disciplinary open access archive for the deposit and dissemination of scientific research documents, whether they are published or not. The documents may come from teaching and research institutions in France or abroad, or from public or private research centers.
L'archive ouverte pluridisciplinaire HAL, est destinée au dépôt et à la diffusion de documents scientifiques de niveau recherche, publiés ou non, émanant des établissements d'enseignement et de recherche français ou étrangers, des laboratoires publics ou privés. 


\title{
On the Self-Organization of Mobile Agents to Ensure Dynamic Multi-level Coverage in Sensor Networks
}

\author{
YOUNES REGRAGUI, LAROSERI, Department of Computer Science, Chouaib Doukkali University \\ NAJEM MOUSSA, LAROSERI, Department of Computer Science, Chouaib Doukkali University
}

A critical issue for the k-coverage problem in wireless sensor networks is how efficiently deploying sensors to cover an area of interest. In many critical scenarios such as in the military field, ensuring that each point in the monitored area of interest is sufficiently covered can guaranty the effectiveness of intrusion detection for both monitoring and tracking applications. Prior research indicated that Mobile Sensor Networks (MSNs) are capable of acting with great flexibility to enhance and cover holes appeared in certain regions when a sensor died due to limited energy and battery lifetime. In this paper, we consider the use of a strategy based on the collective motion mechanisms to relocate sensors nodes to achieve a higher k-coverage level. Each sensor node is able to compare its current k-coverage level with a predefined threshold so as to react dynamically by enabling a specific mobility behavior with a high priority. Based on this mobility behavior, a sensor node can move towards other sensors in its local neighborhood, and it would then be closer enough to them in order to enhance its k-coverage level and then it participates in achieving a higher $\mathrm{k}$-coverage level for the whole group. Simulation results show the effectiveness of our considered approach in terms of the k-coverage level of $30 \%$ as well as a significant improvement in energy consumption.

Additional Key Words and Phrases: Sensor Network, Coverage, k-coverage problem, Sensor Coverage Problem, Collective Motion, Mobile Sensors

ACM Reference Format:

Younes Regragui and Najem Moussa. 2020. On the Self-Organization of Mobile Agents to Ensure Dynamic Multi-level Coverage in Sensor Networks. 1, 1 (February 2020), 12 pages. https://doi.org/10.1145/nnnnnnn.nnnnnnn

\section{INTRODUCTION}

Recently, wireless sensor networks (WSNs) have attracted much higher attention due to its ability to provide different categories of applications for both civil and military fields. In such applications, sensors are organized into groups to cover specific area called the target region and send the sensed data to a remote base station (BS). Efficient coverage in wireless sensor networks (WSNs) is an important issue in the detection of some unexpected events in the area of interest. The coverage problem can emerge when the area of interest is not monitored sufficiently by sensors. For example, for some applications with a higher emergency such as monitoring and tracking applications, each point in the area of interest may need to be

Authors' addresses: Younes Regragui, regragui.y@ucd.ac.ma, LAROSERI, Department of Computer Science, Chouaib Doukkali University, El Jadida, Morocco; Najem Moussa, moussa.n@ucd.ac.ma, LAROSERI, Department of Computer Science, Chouaib Doukkali University, El Jadida, Morocco.

Permission to make digital or hard copies of all or part of this work for personal or classroom use is granted without fee provided that copies are not made or distributed for profit or commercial advantage and that copies bear this notice and the full citation on the first page. Copyrights for components of this work owned by others than ACM must be honored. Abstracting with credit is permitted. To copy otherwise, or republish, to post on servers or to redistribute to lists, requires prior specific permission and/or a fee. Request permissions from permissions@acm.org.

(C) 2020 Association for Computing Machinery.

Manuscript submitted to ACM

Manuscript submitted to ACM 
covered by more than one sensor which is known as K-coverage problem. As each sensor node is expected to die due to a battery drain; and hence coverage holes may appear according to that causing a huge problem of monitoring efficiency. There have been many attempts to solve this problem by identifying how much each sensor node is k-covered by other sensors. For example, authors in 13, to solve the k-coverage sensor deployment problem, they considered two sub-problems: k-coverage placement problem and distributed dispatch problem. The first one is related to the minimum number of sensors required and their locations and the second one asks how to schedule mobile sensors to move to the designated locations when the first problem is resolved. Authors in [12] proposed a similar strategy that considers the problem of placing mobile sensors to get high coverage. Their strategy is based on Voronoi diagrams, where they designed two sets of distributed protocols for controlling the movement of sensors, one favoring communication and one favoring movement. The Voronoi diagrams to detect coverage holes, whereas one of the three proposed algorithms is used to calculate the target locations of sensors if holes exist. In 2], to address this problem, the authors proposed a K-coverage model based on Genetic Algorithm (GA) to extend a WSN lifetime. To identify the optimum active cover, different factors such as targets' positions, the expected consumed energy, and a coverage range of each sensor; are taken into account. Similarly, authors in [3], proposed a new model that uses the Genetic Algorithm (GA) to optimize the coverage in WSNs so as to provide continuous monitoring of specified locations for longest possible time with limited energy resources. In [6], to reflect how well a sensor network is monitored by sensors, the authors proposed a novel solution to determine whether every point in the area of interest is covered by at least $\mathrm{k}$ sensors. They used an approach based on determining how the perimeter of each sensor's sensing range is covered. Each sensor node can execute a polynomial-time algorithm based on location information of its neighbors to measure its level of coverage.

In this paper, we try to resolve this problem by adopting a solution based on a mobility strategy to archive a higher k-coverage level. Our mobility strategy is mainly based on a set of simple rules to allow agents/sensors nodes to move collectively within a group. These rules can govern different movement behaviors such as repulsion, orientation, and attraction. Accordingly, each agent/sensor can continuously compare its current k-coverage level with a predefined threshold ( $\left.\operatorname{cov}_{l e v}\right)$ in order to control its movement according to the needed coverage level by selecting the suitable mobility behavior. The main idea of this paper is that each sensor node in the network to enhance the k-coverage level in its coverage radius, it needs to be closer to other sensor nodes. Each sensor node by enhancing its k-coverage level, in reality, it participates indirectly in establishing a distributed cooperation with all sensor nodes in the network which results in an increased k-coverage level for the whole sensor network. We considered several performance metrics, including the residual energy, the number of sensors nodes still alive, the average normalized velocity and k-coverage level.

The paper is organized as follows. In section 2, our mobility strategy, communication network model and the k-coverage level calculation strategy are presented in detail. In section 3 , the numerical results were reported and the occurrence reason is explained. The conclusions are given in section 4 . 


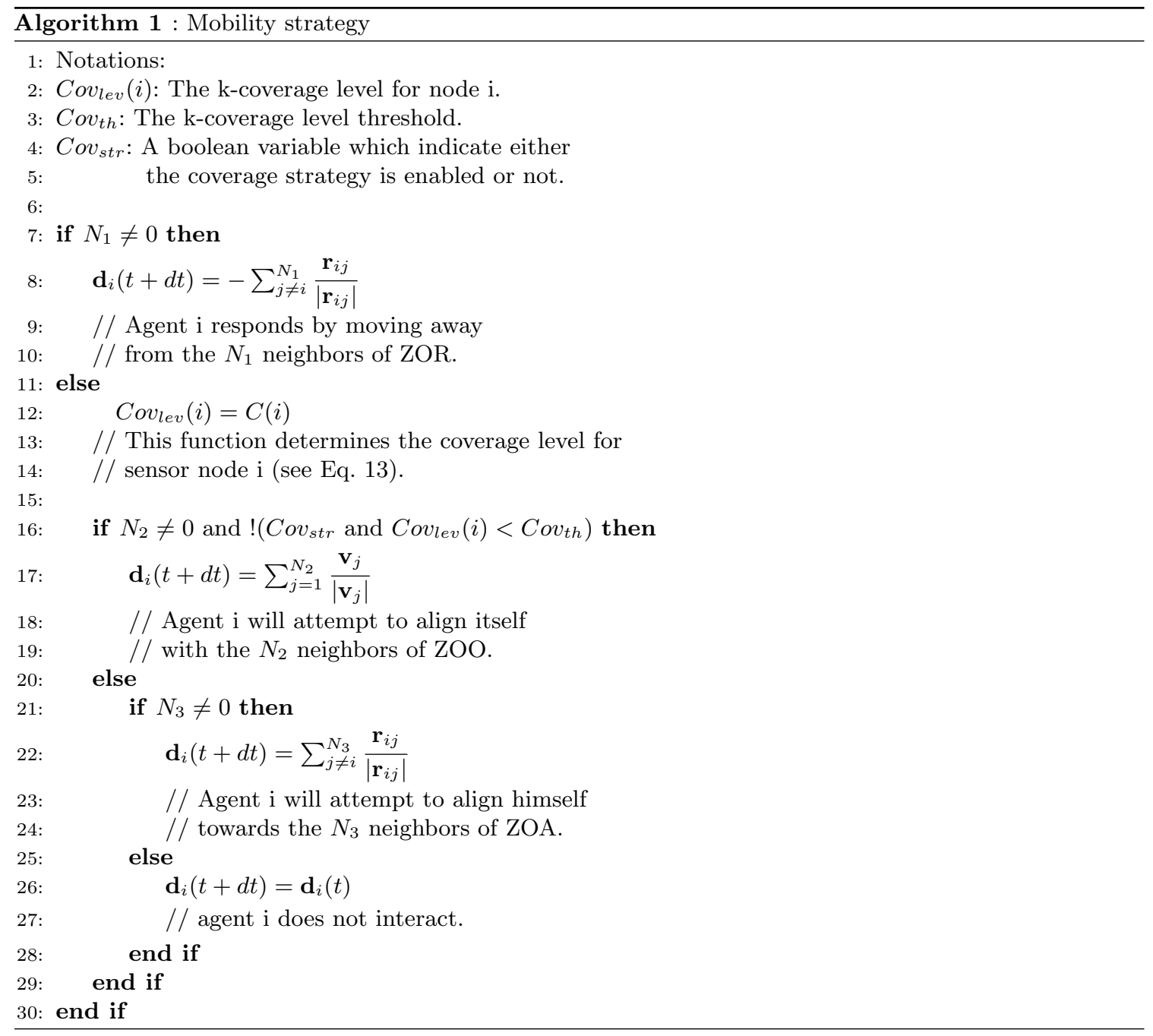

\section{MODEL DESCRIPTIONS}

\subsection{The proposed mobility strategy}

This section describes the considered mobility strategy based on the collective motion of an agents' group and specifically designed for governing the dynamics of agents in the simulation area. It illustrates how autonomous agents are able to make interaction based a superposition of simple rules to define different behaviors with each other, where agents carry out their tasks collectively in order to contribute to a common goal. Accordingly, each agent is able to make interaction with other agents in its neighborhood based on three basic rules [8, 10].

- cohesion: attempt to stay close to each other.

- separation: behavior that avoids collisions by causing an agent to steer away from all of its neighbors. 
- alignment: behavior that causes a particular agent to line up with soldiers close by.

Our mobility strategy is based on a set of behavioral rules capable of providing agents' group to move collectively. Based on this set of rules, our strategy is expected to enhance the k-coverage level as it is based on the selection of the suitable behavioral rule depending on the needed k-coverage level in a sensor node's sensing range. Each agent/sensor node will try to enhance the k-coverage level in its coverage radius until reaching a higher k-coverage level for the whole sensor network.

We consider $N$ agents that move at a constant speed of $v_{0}$ units per second with periodic boundary conditions. Each agent is characterized by his location $\mathbf{c}_{i}(t)$ and velocity $\mathbf{v}_{i}(t)=v_{0} \times \mathbf{d}_{i}(t)$ of direction $\mathbf{d}_{i}(t)$ at time $\mathrm{t}$ 9. In each time step $t$, an agent $i$ assesses the position and/or orientation of neighbors in its local neighborhood within three non-overlapping behavioral zones (see Figure 1) to determine its desired direction of motion $\mathbf{d}_{i}(t+d t)$ at time $t+d t$ based on our algorithm (see Algorithm 1). After that, the agent $i$ moves towards the unit vector $\mathbf{d}_{i}(t+d t)$ that defines the average direction of motion given based on the interaction with other agents within the corresponding zone [10. The unit distance vector from the location point of the agent $i$ in the direction of the agent $j$ is given as follows:

$$
\mathbf{r}_{i j}=\frac{\mathbf{c}_{j}-\mathbf{c}_{i}}{\left|\mathbf{c}_{j}-\mathbf{c}_{i}\right|}
$$

The desired direction of motion at the time $(t+d t)$ is given as follows (see Algorithm 1 ).

After the above process has been performed for each agent $i$, each $i$ agent within the group will move towards the desired direction $d_{i}(t+d t)$ at time $(t+d t)$ as follows:

$$
\mathbf{p}_{i}(t+d t)=\mathbf{p}_{i}(t)+\mathbf{d}_{i}(t+d t) \times v_{0} \times d t
$$

\subsection{Wireless communication network model}

In this section, we consider that each agent/sensor node is capable of communicating using short-range radio transmissions. Also, each agent is capable of sensing and generating periodically data packets that will be

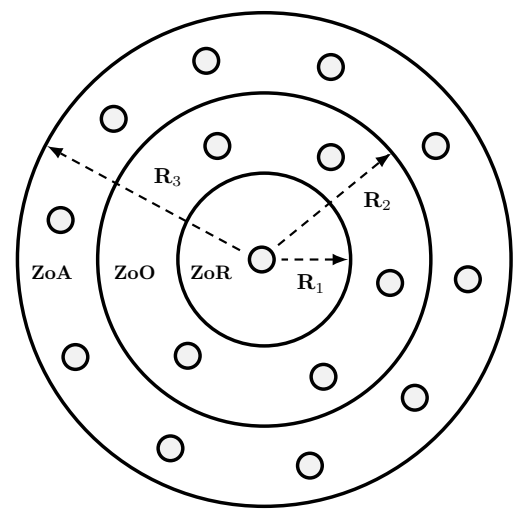

Fig. 1. Representation of a sensor node in our mobility strategy with deferent behavioral zones: zor=zone of repulsion, $\mathrm{zoo}=$ zone of orientation, $\mathrm{zoa}=$ zone of attraction. 


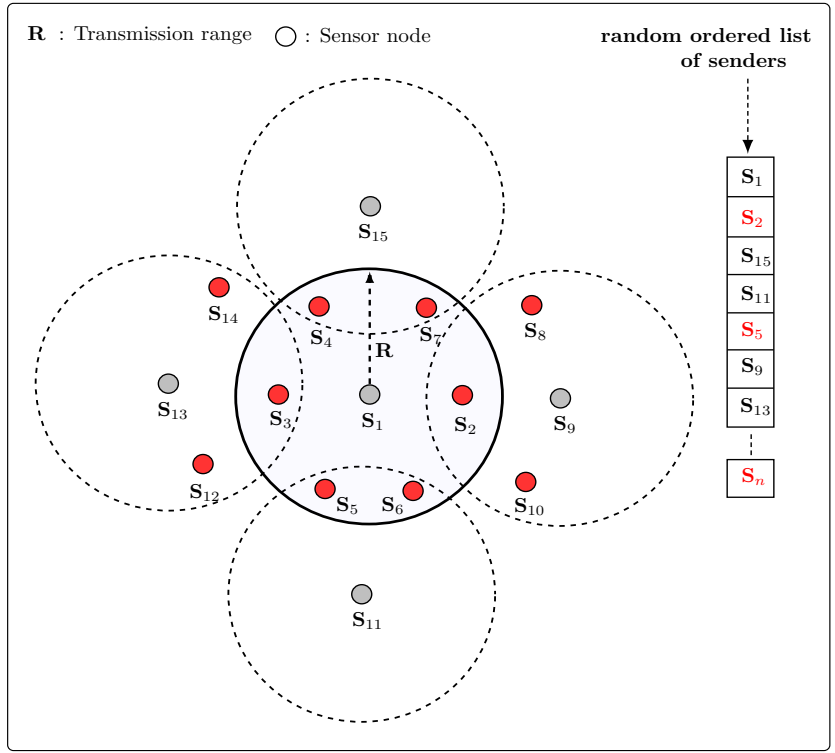

Fig. 2. Illustration example of multiple-access to wireless channel based on the proposed Mac protocol.

transmitted to a mobile sink node based on a simple mac protocol and a probabilistic flooding algorithm [7, 11. The sink node is selected randomly at the beginning of the simulation. For each pair of transmitter and receiver $\{i, j\}$ located within the transmission range $\mathrm{R}$ of each other, the received signal strength at node $\mathrm{j}$ decays with the distance between them according to Friss free space model [5] which defines the relationship between the transmit power of node $i$ and the received signal power by node $\mathrm{j}$ in terms of the distance between them. The received signal power by node $\mathrm{j}$ is given as follows:

$$
\mathbf{p}_{r}(j)=\frac{p_{t}(i)}{\sigma d_{i, j}^{\alpha}}
$$

where $d_{i, j}$ is the Euclidean distance between agent $\mathrm{i}$ and $\mathrm{j}, p_{i}$ and $p_{j}$ are the transmitted power and the received power, respectively, and $\sigma=\frac{(4 \pi)^{2}}{G_{t} G_{r} \lambda^{2}} \quad[5$. We use Friss free space channel propagation model [5] (i.e., $\alpha=2$ ). Thus, to transmit a l-bit message to node $\mathrm{j}$ located a distance $r_{i, j}$ from node $\mathrm{i}$, the radio of node i expends:

$$
\mathbf{E}_{T x}(l, r)=E_{\text {ele }} l+\epsilon_{f s} l r^{2}
$$

Accordingly, the required transmit power is given as follows:

$$
\mathbf{p}_{t}(i)=\epsilon_{f s} l r^{2}
$$

where $r$ is the transmission range of node i. 
The radio of node $\mathrm{j}$, to receive the transmitted message from node $\mathrm{i}$, expends:

$$
\mathbf{E}_{R x}(l, r)=E_{\text {ele }} l
$$

As we consider a periodic data gathering. The energy to sense data of 1 bits by sensor node i at each time period is given as follows:

$$
\mathbf{E}_{\text {Sens }}(i)=\alpha^{\prime} l
$$

On the other hand, we have assumed that each data packet sent by node $\mathrm{i}$ to node $\mathrm{j}$ is received successfully if the signal power received by node $\mathrm{j}$ is strong enough compared to the thermal noise and the sum of the interference power received from other transmitters. This can be written formally as:

$$
\mathbf{S I N R}=\frac{\left(p_{t}(i) / \sigma d_{i, j}^{\alpha}\right)}{N_{0}+\sum_{k \neq i, j}^{N}\left(p_{t}(k) / \sigma d_{k, j}^{\alpha}\right)} \geq \beta
$$

where SINR is the signal-to-noise-plus-interference ratio, $\beta$ is the SINR threshold requirement for successful communication, $N_{0}$ represents the thermal noise floor and the receiver noise figure, its value equals to $-82 \mathrm{dBm}[4], d_{i, j}$ is the distance between node $\mathrm{i}$ and $\mathrm{j}$. The term $\sum_{k \neq i, j}^{N}\left(p_{t}(k) / \sigma d_{k, j}^{\alpha}\right)$ is the interference contribution from $\mathrm{k}$ sender nodes within the transmission range of the receiving node $\mathrm{j}$.

\subsection{Implementation of Mac protocol}

Here, a simplified Mac protocol is implemented to solve the problem of multiple-access of different transmitters to a wireless channel at the same time. So, our proposed Mac protocol can be used to control the multipleaccess of transmitters by limiting the channel assignment only for some transmitters in the network in the following way: At each time step t, we create a randomly ordered list of transmitters. We go through this list, where for example, the first transmitter node $k_{0}$ on the list gets access to the wireless channel and all transmitters which may cause interference with $k_{0}$ will be blocked for time step $t$, including only those located in the transmission range of $k_{0}$ (see Figure 2). This procedure is repeated for each transmitter in the list if it is still authorized to access the channel. Figure 2 shows that all senders nodes located in the transmission range of sender $\mathrm{S}_{1}$ (e.g., $\mathrm{S}_{2}, \mathrm{~S}_{3}, \mathrm{~S}_{4}, \mathrm{~S}_{5}, \mathrm{~S}_{6}$ and $\mathrm{S}_{7}$ ) are blocked at time step t, where only $\mathrm{S}_{1}$ is allowed to broadcast a packet. The total number of senders authorized to access the channel in the randomly ordered list at time step $\mathrm{t}$ are marked with a gray color, whereas blocked senders are marked with a red color (e.g., $\mathrm{S}_{1}, \mathrm{~S}_{15}, \mathrm{~S}_{11}, \mathrm{~S}_{9}$ and $\mathrm{S}_{13}$ ).

\subsection{The calculation of $\mathbf{k}$-coverage level}

In the section, we propose a solution to the k-UC problem which extends a previous solution in [6] with a different approach. Our calculation approach of the k-coverage level is based on a set of simple steps in computational geometry and mathematics. For this purpose, we consider that all sensor nodes have the same sensing range, our proposed strategy for the measurement of the k-coverage level is also considered to be a solution to the k-UC problem. In this paper, we consider determining whether a sensor is k-perimeter-covered or not. We can say that any sensor $s_{i}$ is k-perimeter-covered if a segment of $s_{i}$ 's perimeter is k-perimetercovered by at least k sensors. Consider two sensors $s_{i}$ and $s_{j}$, a sensor node $s_{i}$ is perimeter-covered by sensor $s_{j}$ when $d\left(s_{i}, s_{j}\right) \leq 2 r$, where $d\left(s_{i}, s_{j}\right)$ is the euclidian distance (see Figure 3). Accordingly, $\alpha$ is given as 
follows:

$$
\alpha=\arccos \left(\frac{d\left(s_{i}, s_{j}\right)}{2 r}\right)
$$

Then the arch of $s_{i}$ falling in the angle $\phi_{i, j}=[\pi-\alpha, \pi+\alpha]$ represents the $s_{i}$ 's perimeter-covered by $s_{j}$ and $\pi$ is the angle between 0 and the segment $d\left(s_{i}, s_{j}\right)$. Now, we consider a small angle $\delta \theta \in[0,2 \pi]$ that defines a small segment of $s_{i}$ 's perimeter. To determine if $\delta \theta$ intersects with the angle $\phi_{i, j}$ or not, we define the function $\mathbf{f}\left(\phi_{i, j}, \delta \theta_{k}\right)$ as:

$$
\mathbf{f}\left(\phi_{i, j}, \delta \theta_{k}\right)= \begin{cases}1 & \text { if }\left(\phi_{i, j} \cap \delta \theta_{k}=\delta \theta\right) \\ 0 & \text { Otherwise. }\end{cases}
$$

where $\sum_{k=1}^{n} \delta \theta_{k}=2 \pi, \mathrm{n}$ is the number of angle intervals $\delta \theta_{k}$. The value of $\mathrm{n}$ is given as follows:

$$
n=E\left(\frac{2 \pi}{\delta \theta}\right)
$$

We define an other function $g_{i, k}\left(\delta \theta_{k}, m\right)$ that determines the number of sensors $s_{j}$ 'perimeters which intersect with the angle $\delta \theta_{k}$ as follows:

$$
\mathbf{g}_{i, k}\left(\delta \theta_{k}, m\right)=\sum_{j=1, j \neq i}^{m} f\left(\phi_{i, j}, \delta \theta_{k}\right)
$$

where $0 \leq g_{i, k}\left(\delta \theta_{k}, m\right) \leq m$ and $\mathrm{m}$ is the number of neighbor sensors of sensor $s_{i}$. Then the k-coverage level of $s_{i}$ ' perimeter by $m$ neighbor sensors can be derived as:

$$
\mathbf{C}(i)=\min \left(\left\{g_{i, 1}, g_{i, 2}, \ldots, g_{i, n}\right\}\right)
$$

Here, we like notify that as more as $\delta \theta$ is very small, we get more precision for the measurement of the k-coverage level.

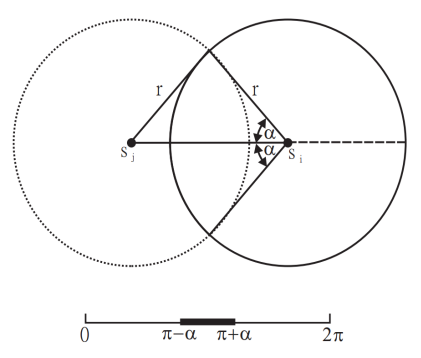

a)

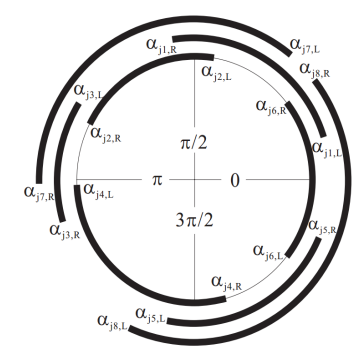

b)

Fig. 3. Illustration example of 1-perimeter-covered sensor (a) and k-perimeter-covered sensor node (b) [6].

\subsection{Queue Management}

In the following part, we separate the forwarding and originating packets into two queues. As sensor nodes are performing a periodically gathering of data, at each sensing time interval, a new packet is added to the originating queue if this one is still empty. In addition, we use a very simple policy that manages the priority between originating and forwarding data packets when both queues are not empty. Then, only one among 
the two queues is selected with the same probability when the sensor node gets access to the channel for sending a data packet.

\section{SIMULATION ENVIRONMENT}

In this paper, all models have been implemented using $\mathrm{C}++$ language, where a sensor network of $\mathrm{N}$ mobile nodes (agents) deployed close to each other in a simulation area of $200 \times 200 \mathrm{~m}^{2}$. At each time step $d t$. The agents/nodes are able to send data packets towards the sink node. The considered simulation parameters in this work are given in the table below 1 .

Table 1. Parameters used in the simulation.

\begin{tabular}{|l||l||l|}
\hline Parameter & Symbol & Value \\
\hline \hline Simulation area & - & $200 \mathrm{~m} \times 200 \mathrm{~m}$ \\
Simulation time & - & $8000(\mathrm{~s})$ \\
Zone of repulsion & $\mathrm{N}$ & 100 \\
Zone of orientation & $Z o R$ & $1 \mathrm{~m}$ \\
Zone of attraction & $Z o O$ & $10 \mathrm{~m}$ \\
Initial velocity of nodes & $v_{0}$ & $50 \mathrm{~m}$ \\
Transmission range & $\mathrm{r}$ & $1(\mathrm{~m} / \mathrm{s})$ \\
Mobility time step & $d t$ & $50 \mathrm{~m}$ \\
Communication time step & $d t^{\prime}$ & $1(\mathrm{~s})$ \\
Initial energy & $E_{0}$ & $0.1(\mathrm{~s})$ \\
Electronics energy & $E_{\text {ele }}$ & $50 \mathrm{~nJ} / \mathrm{bit}$ \\
Transmitter Amplifier & $\epsilon_{f s}$ & $10 \mathrm{pJ} / \mathrm{bit} / \mathrm{m}^{2}$ \\
Packet size & $\mathrm{l}$ & $4000 \mathrm{bits}$ \\
Sensed data interval & $S e n s_{i n t}$ & $1(\mathrm{~s})$ \\
Sense energy per bit & $\alpha^{\prime}$ & $60 \mathrm{~nJ} / \mathrm{bit}$ \\
K-coverage threshold & cov lev & 2 \\
\hline
\end{tabular}

\subsection{Performance Metrics}

Several performance metrics are developed to evaluate and analyze the k-coverage level of a sensor network. We monitor the behavior of the sensor network in terms of k-coverage level, residual energy, number of sensor nodes alive and the average normalized velocity.

- Average normalized velocity: we define the Average normalized velocity as the degree of alignment of group' agents within the group. It increases as the degree of alignment group' agents within the group increases [1]. The average normalized velocity of the group' agents is then given as follows:

$$
\boldsymbol{V}_{n}(t)=\frac{1}{v_{0} N}\left|\sum_{i=1}^{N} \mathbf{v}_{i}(t)\right|
$$

Manuscript submitted to ACM 


\section{SIMULATION RESULTS}

In this section, we analyzed the performance of our proposed strategy in two cases: with and without a strategy. We consider that all sensor nodes have limited energy of $0.5 \mathrm{~J}$ except for the sink which has unlimited energy resources, accordingly we do not account the energy spent by the sink. In order to evaluate sensor network condition, several performance metrics are considered, including the energy consumption, the number of sensor nodes still alive, average normalized velocity and the k-coverage level. The simulation is performed based on our simulator implemented in $\mathrm{C}++$ language and the results are averaged over 200 replications. Wireless communications and coverage strategy are enabled after a certain time interval in order to allow group' agents to make enough interactions to reach a higher group cohesion. After this time interval, all models are enabled for a time interval equals to $8000 \mathrm{~s}$.

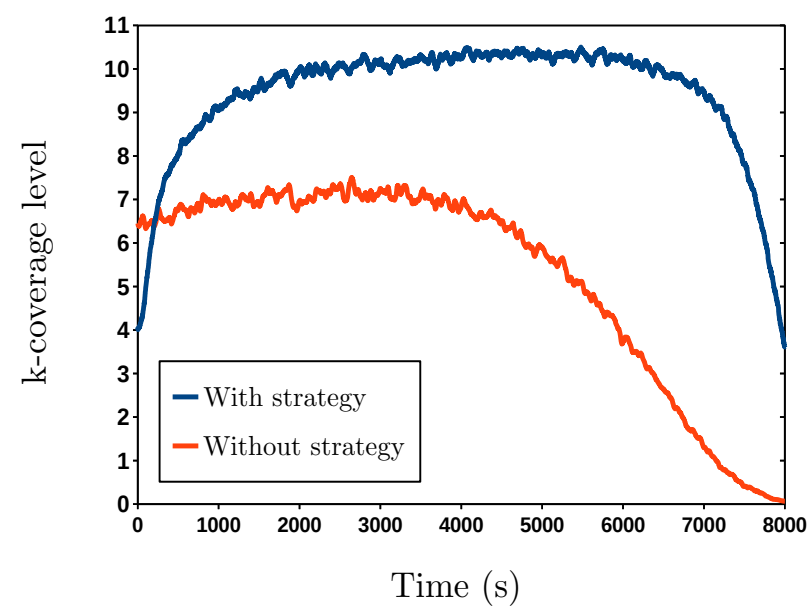

Fig. 4. The change in k-coverage level versus time.

Figure 4 shows that the k-coverage level illustrated by our proposed coverage strategy is increased significantly when the strategy is enabled as compared with the case where the strategy is absent. After a phase transition $\in[1,2000] s$, the coverage level remains almost stable and equals to $(\simeq 10)$. When the coverage strategy is absent, it is observed that the coverage level is stabilized at only $(\simeq 7)$. This means that our strategy shows an improvement of $\simeq 30 \%$ of the k-coverage level. This can be explained by the fact that the increase in k-coverage level is due mainly to a decrease in the nearest distance between sensor nodes as each one tries to enhance its k-coverage level by performing a supplementary attraction with their neighbors. Moreover, this also means that the increase in k-coverage level is combined with a decrease in the total covered area as agents' group becomes less elongated due to an increase in the group cohesion. The decrease in the total covered area can be considered to be a worse problem in some cases depending on the considered scenario. For example, if we consider a scenario where a mobile sensor network is built to accomplish some missions such as tracking targets by following a goal trajectory. In this case, it is possible to solve this problem by varying the number of sensor nodes or mobility' parameters depending on needed coverage area while following this trajectory. In addition, it is observed that a significant improvement in 


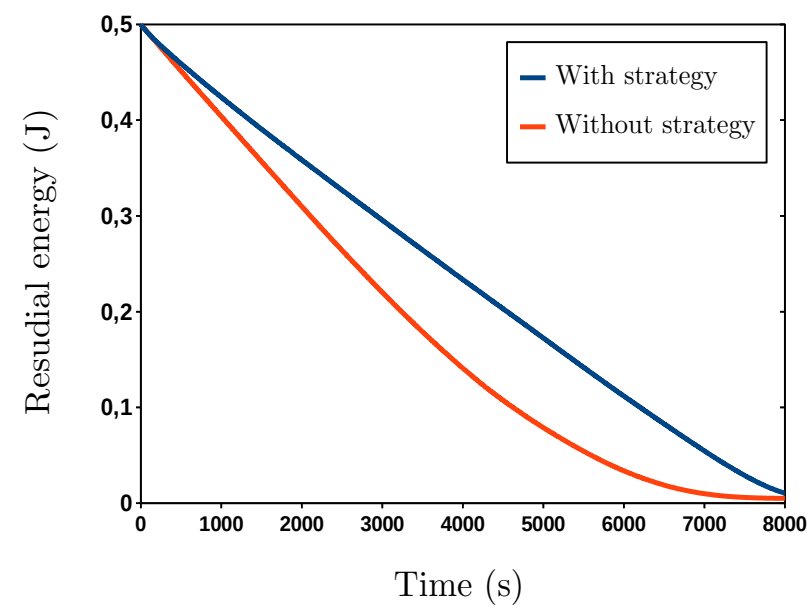

Fig. 5. The change in residual energy versus time.

both the residual energy and the number of sensor nodes still alive (see Figure 5 and Figure 6). This is due to the decrease in the distance between group' agents which means that each data packet can reach the destination with an only small number of hops and a low forwarding rate.

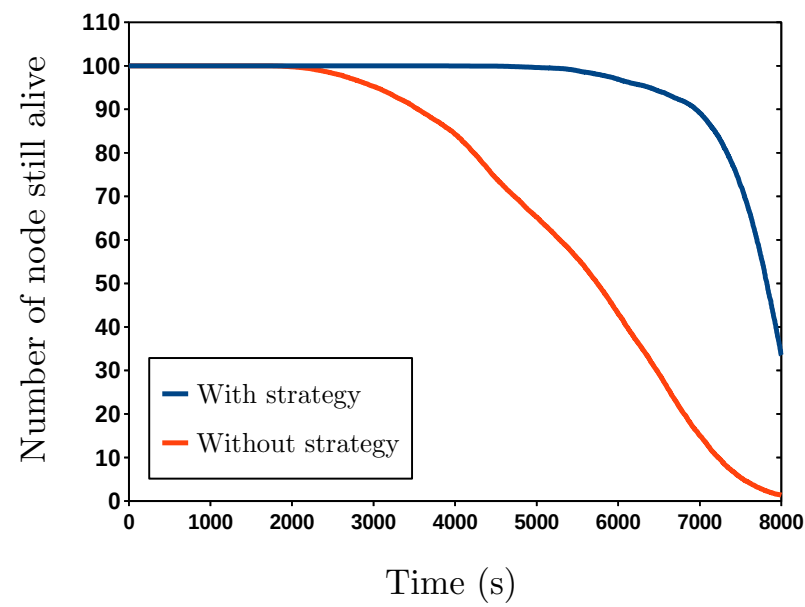

Fig. 6. The change in number of sensor nodes still alive versus time.

This means that when group' agents are closer enough to each others, each sent packet is received by many nodes, and therefore a small number of nodes which receive this packet for the first time and will try to forward it. Therefore, the reduction in the rebroadcasting rate has a significant impact on energy consumption. On the other hand, it is observed that the coverage strategy has a significant impact on the average normalized velocity, where it is decreased significantly as compared with results without a strategy (see Figure 7). In contrast, when the strategy is absent, the average normalized velocity reaches a higher Manuscript submitted to ACM 
level in $[1,2500] \mathrm{s}$ as the number of sensor nodes still alive is higher. With the increase of time, the average normalized velocity starts to decrease significantly proportionally with the increase in the residual energy and the number of sensor nodes still alive. The decrease in the average normalized velocity means that group' agents are not moving with parallel alignment (e.g., they does not move towards a specific direction). The lack of accuracy concerning the group's direction can have a worse impact for some critical missions such as tracking a target in real-time, where group' agents are following a specific trajectory.

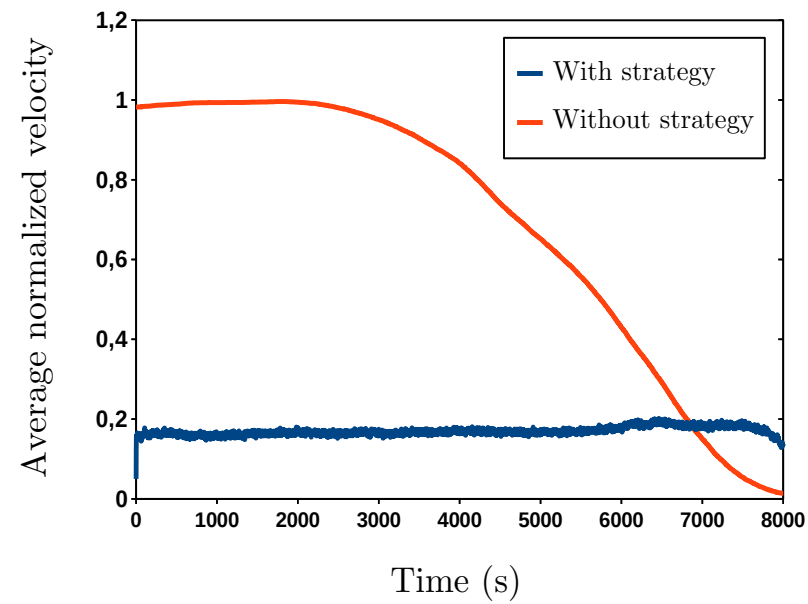

Fig. 7. The change in average normalized velocity versus time.

\section{CONCLUSIONS}

In this paper, we have provided an analysis of a mobile sensor network in terms of the k-coverage level and other performance metrics such as residual energy, the number of sensor nodes still alive and the average normalized velocity. We considered a dynamic coverage strategy based on the collective motion mechanisms to enable agents to move collectively based on a set of simple rules responsible for different behavioral rules within the group. The considered collective motion strategy is extended to take into consideration the k-coverage level for every sensor node in the network. Accordingly, each sensor node can compare its current k-coverage level with a predefined threshold so as to attempt to align itself towards their neighbors to enhance its k-coverage level. While a sensor node tries to increase its k-coverage level, in reality, it participates indirectly to increase the k-coverage level of the whole sensor network. Our strategy shows a significant improvement in the k-coverage level as compared with obtained results when the coverage strategy is absent. Moreover, our strategy illustrates an improvement in terms of the energy consumption due to a decrease in the distance between group' agents which directly has an impact on the decrease in the rate of data packet forwarding. Indeed, the obtained results show that the increase in attraction behavior has a significant impact on group alignment. This issue can have a worse impact in the case of some critical scenarios where the group' agents must follow a precise trajectory with less uncertainty towards a specific target. This point can be considered as future work. We will provide a supplementary improvement of our strategy to support intelligent behavioral rules that can take into account the accuracy of group motion. 


\section{REFERENCES}

[1] I. D. Couzin, J. Krause, R. James, G. D. Ruxton, and N. R. Franks. Collective memory and spatial sorting in animal groups. Journal of theoretical biology, 218(1):1-11, 2002.

[2] M. Elhoseny, A. Tharwat, A. Farouk, and A. E. Hassanien. K-coverage model based on genetic algorithm to extend wsn lifetime. IEEE sensors letters, 1(4):1-4, 2017.

[3] M. Elhoseny, A. Tharwat, X. Yuan, and A. E. Hassanien. Optimizing k-coverage of mobile wsns. Expert Systems with Applications, 92:142-153, 2018.

[4] W. B. Heinzelman. Application-specific protocol architectures for wireless networks. PhD thesis, Massachusetts Institute of Technology, 2000.

[5] W. B. Heinzelman, A. P. Chandrakasan, and H. Balakrishnan. An application-specific protocol architecture for wireless microsensor networks. IEEE Transactions on wireless communications, 1(4):660-670, 2002.

[6] C.-F. Huang and Y.-C. Tseng. The coverage problem in a wireless sensor network. Mobile networks and Applications, 10(4):519-528, 2005.

[7] H. Lim and C. Kim. Flooding in wireless ad hoc networks. Computer Communications, 24(3-4):353-363, 2001.

[8] Y. Regragui and N. Moussa. Agent-based system simulation of wireless battlefield networks. Computers ES Electrical Engineering, 56:313-333, 2016.

[9] Y. Regragui and N. Moussa. Simulation of wireless sensors in collective motion. In 2018 6th International Conference on Wireless Networks and Mobile Communications (WINCOM), pages 1-6. IEEE, 2018.

[10] T. Vicsek and A. Zafeiris. Collective motion. Physics Reports, 517(3):71-140, 2012.

[11] K. Viswanath and K. Obraczka. Modeling the performance of flooding in wireless multi-hop ad hoc networks. Computer Communications, 29(8):949-956, 2006.

[12] G. Wang, G. Cao, and T. F. La Porta. Movement-assisted sensor deployment. IEEE Transactions on Mobile Computing, $5(6): 640-652,2006$.

[13] Y.-C. Wang and Y.-C. Tseng. Distributed deployment schemes for mobile wireless sensor networks to ensure multilevel coverage. IEEE Transactions on Parallel and Distributed Systems, 19:1280-1294, 2008. 